\title{
Standard model with spontaneously broken quantum scale invariance
}

\author{
D. M. Ghilencea, ${ }^{1,2, *}$ Z. Lalak, ${ }^{3, \dagger}$ and P. Olszewski ${ }^{3, *}$ \\ ${ }^{1}$ Theoretical Physics Department, National Institute of Physics and Nuclear Engineering, \\ Bucharest 077125, Romania \\ ${ }^{2}$ Theory Division, CERN, 1211 Geneva 23, Switzerland \\ ${ }^{3}$ Institute of Theoretical Physics, Faculty of Physics, University of Warsaw, 5 Pasteura Street, \\ 02-093 Warsaw, Poland
}

(Received 28 July 2017; published 25 September 2017)

\begin{abstract}
We explore the possibility that scale symmetry is a quantum symmetry that is broken only spontaneously and apply this idea to the standard model. We compute the quantum corrections to the potential of the Higgs field $(\phi)$ in the classically scale-invariant version of the standard model ( $m_{\phi}=0$ at tree level) extended by the dilaton $(\sigma)$. The tree-level potential of $\phi$ and $\sigma$, dictated by scale invariance, may contain nonpolynomial effective operators, e.g., $\phi^{6} / \sigma^{2}, \phi^{8} / \sigma^{4}, \phi^{10} / \sigma^{6}$, etc. The one-loop scalar potential is scale invariant, since the loop calculations manifestly preserve the scale symmetry, with the dimensional regularization subtraction scale $\mu$ generated spontaneously by the dilaton vacuum expectation value $\mu \sim\langle\sigma\rangle$. The Callan-Symanzik equation of the potential is verified in the presence of the gauge, Yukawa, and the nonpolynomial operators. The couplings of the nonpolynomial operators have nonzero beta functions that we can actually compute from the quantum potential. At the quantum level, the Higgs mass is protected by spontaneously broken scale symmetry, even though the theory is nonrenormalizable. We compare the oneloop potential to its counterpart computed in the "traditional" dimensional regularization scheme that breaks scale symmetry explicitly $(\mu=$ constant) in the presence at the tree level of the nonpolynomial operators.
\end{abstract}

DOI: $10.1103 /$ PhysRevD.96.055034

\section{MOTIVATION}

In this paper, we explore the idea that scale symmetry is a quantum symmetry and study its implications for physics beyond the standard model (SM). However, this symmetry is broken in the real world. We shall consider here only spontaneous breaking of this (quantum) symmetry. ${ }^{1}$ One motivation of our study is that scale symmetry plays a role in the UV behavior of the models. In particular, the SM with a classical Higgs mass parameter $m_{\phi}=0$ has an increased symmetry: it is scale invariant at the tree level; this was invoked [1] to protect $m_{\phi}$ naturally [2] from large quantum corrections, but a full quantum study is needed.

Consider a classically scale-invariant theory. One known issue when studying scale symmetry at the quantum level is that the regularization of the loop corrections introduces a dimensionful parameter (subtraction scale $\mu$ )

\footnotetext{
*dumitru.ghilencea@cern.ch

zygmunt.lalak@fuw.edu.pl

\#pawel.olszewski@fuw.edu.pl

${ }^{1}$ By quantum scale symmetry, we mean that the full oneparticle irreducible (1PI) quantum action is scale invariant.

Published by the American Physical Society under the terms of the Creative Commons Attribution 4.0 International license. Further distribution of this work must maintain attribution to the author(s) and the published article's title, journal citation, and DOI.
}

that breaks explicitly the scale symmetry that we want to investigate. ${ }^{2}$ To avoid such breaking, the UV regularization must preserve this symmetry. This is done by using a subtraction function $\mu(\sigma)$, which generates (dynamically) a subtraction scale $\mu(\langle\sigma\rangle)$ when the field $\sigma$ acquires a vacuum expectation value (vev) $\langle\sigma\rangle$ after spontaneous scale symmetry breaking. For details on this, see Ref. [3] and recent examples at one loop [4-8] and higher loops $[9,10]$. Here, $\sigma$ is the Goldstone mode (dilaton) of the spontaneously broken scale symmetry ${ }^{3}$ and is an additional degree of freedom of the theory.

The model we consider is a scale-invariant SM, defined as the SM with classical $m_{\phi}=0$ and extended by the dilaton. The goal is to use this scale-invariant regularization to compute quantum corrections to the scalar potential. The quantum result is scale invariant, so it can only have spontaneous scale symmetry breaking, with a flat direction for the dilaton $(\sigma)$. For clarity, this result is then compared to that in the "usual" dimensional regularization (DR) of $\mu=$ constant scale, which breaks explicitly the scale symmetry at the quantum level.

\footnotetext{
${ }^{2}$ One could use a regularization that does not keep manifest scale symmetry and then attempt to restore it "by hand" at the end, but this misses scale-invariant operators if the theory is nonrenormalizable (see later, Sec. II).

${ }^{3}$ To be exact, the mass eigenstates may actually contain a small mixing of original $\phi, \sigma$.
} 
Let us consider first a simplified scale-invariant (classical) theory (e.g., Refs. [11-27]) of two real scalar fields $\phi$ (Higgs-like) and $\sigma$. The potential $V$ is a homogeneous function, having no dimensionful couplings, so

$V(\phi, \sigma)=\sigma^{4} W(\phi / \sigma)$, where $W(\phi / \sigma)=V(\phi / \sigma, 1)$.

We assume that $V(\phi, \sigma)$ has spontaneous scale symmetry breaking i.e., that $\sigma$ acquires a nonzero vacuum expectation value $\langle\sigma\rangle \neq 0$. We thus search for such a solution and for the necessary condition for this spontaneous breaking to happen. With $\langle\sigma\rangle \neq 0$, it is then easy to see that the minimum conditions $V_{\sigma}=V_{\phi}=0\left(V_{\alpha}=\partial V / \partial \alpha\right)$ are equivalent to

$$
W(\rho)=W^{\prime}(\rho)=0, \quad \rho \equiv \phi / \sigma .
$$

These equations can have a common solution $\rho_{0} \equiv\langle\phi\rangle /\langle\sigma\rangle$, if the couplings satisfy a particular condition (constraint); see below. Then, a flat direction exists in the plane $(\phi, \sigma)$ with $\phi=\rho_{0} \sigma$. Indeed, if $(\langle\phi\rangle,\langle\sigma\rangle)$ is a ground state with $V=0$, then so is $(t\langle\phi\rangle, t\langle\sigma\rangle), t$ real. Also, the second derivatives matrix $V_{\alpha \beta}$ with respect to $\alpha, \beta=\phi, \sigma$ has $\operatorname{det}\left(V_{\alpha \beta}\right) \propto\left(4 W W^{\prime \prime}-3 W^{\prime 2}\right)=0$ on the ground state, so a massless state is indeed present corresponding to the flat direction. Finally, since $\rho_{0}$ is a root of both $W$ and of its derivative $W^{\prime}$, then $W(\phi / \sigma) \propto\left(\phi / \sigma-\rho_{0}\right)^{2}$, while if $V$ depends only on even powers of the scalar fields (our model below), then the general structure is

$$
W(\phi / \sigma) \propto\left(\phi^{2} / \sigma^{2}-\rho_{0}^{2}\right)^{2} .
$$

Note that the vanishing vacuum energy $V(\langle\phi\rangle,\langle\sigma\rangle)=0$ follows from the (spontaneously broken) scale symmetry; see Eq. (2). A scale-invariant regularization of this theory leads to a scale-invariant quantum potential, which thus remains of the form shown in Eq. (1). Hence, the above discussion around Eqs. (1), (2), and (3) remains true at the quantum level, including the possibility of spontaneousonly breaking of the scale symmetry.

One of the two minimum conditions in (2) fixes the ratio $\rho_{0}=\langle\phi\rangle /\langle\sigma\rangle$ in terms of the (dimensionless) couplings of the theory. Thus, all vevs of such a theory, including $\langle\phi\rangle$, are proportional to $\langle\sigma\rangle \neq 0$, which is a(n) (unknown) parameter of the theory. The second minimum condition, after eliminating $\rho_{0}$ between the two equations in (2), gives a relation among the couplings of the theory in the order of perturbation in which $V$ is computed. This means that one coupling, say $\lambda_{\sigma}$ (the dilaton self-coupling), is defined in terms of the rest, $\lambda_{\sigma}=f\left(\lambda_{j \neq \sigma}\right)$. This relation follows from demanding that $V$ has a flat direction [26], which is a consequence of our requiring that quantum scale symmetry be broken spontaneously. Such relation can be "arranged" by one initial classical tuning, with subsequent (quantum) tunings bringing "acceptable" $\mathcal{O}\left(\lambda_{j}\right)$ corrections to this relation, relative to the previous perturbation $\operatorname{order}^{4}$; this tuning ensures a vanishing vacuum energy $V(\langle\phi\rangle,\langle\sigma\rangle) \sim$ $W\left(\rho_{0}\right)=0$ [see conditions (2)].

We stress that the above picture, which builds on previous studies [3-10], is very different from that obtained in the "traditional" DR scheme ( $\mu=$ constant scale) that is often used in classically scale-invariant models, e.g., Refs. [18-27]; in such models, scale symmetry is broken explicitly by the (regularization of) quantum effects, and then conditions (1) and (2) are not true anymore at the quantum level, and the flat direction is lifted by quantum corrections (the dilaton is then a pseudo-Goldstone, which is light).

What about the hierarchy problem? In the absence of gravity (not included here), the standard model has no hierarchy problem. However, this situation is no longer true under the reasonable assumption that there is some "new physics" beyond SM, e.g. a large vev of a new scalar field that couples to Higgs, etc. In the model we consider, defined by the scale-invariant version of the SM extended by the dilaton, we have "new physics" beyond the SM, represented by the vev $\langle\sigma\rangle$ that spontaneously breaks the scale symmetry. $\langle\sigma\rangle$ can be very large compared to $\langle\phi\rangle$, which fixes the electroweak scale. In the Brans-DickeJordan theory of gravity, not considered here, one expects ${ }^{5}$ $\langle\sigma\rangle \sim M_{\text {Planck }}$. $(\langle\sigma\rangle$ can then be regarded as a physical cutoff of the theory.) We simply enforce such hierarchy by choosing a very weak coupling of the visible to the hidden sector of the dilaton ${ }^{6}$ [31]. Such hierarchy is, however, stable under quantum corrections, so $m_{\phi} \sim\langle\phi\rangle \ll\langle\sigma\rangle$ without tuning at the quantum level $[4,8]$, and we verify this in our model at one loop. This is expected to remain true to all orders in perturbation theory since scale symmetry is preserved by the regularization and is broken only spontaneously. ${ }^{7}$ We thus have an example of a quantum stable hierarchy, with a vanishing vacuum energy at the loop level, that follows from the demand of spontaneously broken quantum scale symmetry.

\footnotetext{
${ }^{4}$ Perturbativity, $\lambda_{\sigma}<1$, is maintained for a weak coupling $\lambda_{m}$ between the visible $(\phi)$ and hidden $(\sigma)$ sectors; see later, Eq. (9) (a) or (11) (a), which fixes $\lambda_{\sigma}<1$ in terms of the other couplings, for a small enough $\lambda_{m}$.

${ }^{5}$ Such hierarchy can be generated dynamically (Refs. [28,29] or as in Ref. [30]).

${ }^{6}$ One takes $\left|\lambda_{m}\right| \ll \lambda_{\phi} ; \lambda_{m}$ : coupling of hidden $(\sigma)$ to the visible sector $(\phi) ; \lambda_{\phi}$ : Higgs self-coupling; see later in the paper, Eq. (7). The hierarchy of vevs (scales) is then replaced by a (more fundamental) hierarchy of dimensionless couplings.

${ }^{7}$ Scale symmetry may also be broken at some high scale due to Landau poles of some of the couplings of the theory or due to other nonperturbative effects. We do not consider these effects here since they involve physics above Planck scale in which case the present flat space-time picture is not appropriate-one needs to upgrade this formalism to include Brans-Dicke-Jordan gravity; see, e.g., Ref. [28].
} 
In the following, we apply these ideas to the scaleinvariant version of the SM (with classical Higgs mass $m_{\phi}=0$ ) extended by the dilaton. The Higgs and the dilaton have a potential dictated solely by the classical scale symmetry, so it can contain higher-dimensional nonpolynomial operators such as $\phi^{6} / \sigma^{2}, \phi^{8} / \sigma^{4}$, etc. We then compute the one-loop potential with a scale-invariant regularization, so a flat direction is maintained at the quantum level. Even if the tree-level potential does not include the nonpolynomial operators (by tuning their couplings to 0 ), they are generated at one loop with finite coefficient [8] or as two-loop or higher counterterms $[9,10]$ - this means the scale-invariant quantum theory is nonrenormalizable. Further, the quantum consistency of the theory is shown by verifying the Callan-Symanzik equation of the potential in the presence of the nonpolynomial effective operators, gauge and Yukawa interactions. We also compare the scaleinvariant one-loop potential to its counterpart computed in the usual DR scheme that breaks scale symmetry explicitly ( $\mu=$ constant), in the presence of these effective operators at tree level.

If scale symmetry is preserved by one-loop $V$, there is no dilatation anomaly that is a result of explicit scale symmetry breaking by quantum calculations with $\mu=$ constant. Contrary to common lore, the couplings still run with momentum [6-8] since the vanishing of the beta functions is not a necessary condition for scale invariance. Their oneloop running is identical to that in the theory with explicit scale symmetry breaking ( $\mu=$ constant), but at two loops, they start to differ in theories with spontaneous vs explicit breaking $[7,10]$.

This analysis in flat space-time should be extended to include the effects of gravity that we ignored. Since Einstein gravity breaks scale symmetry, a natural setup to include such effects is to consider the Brans-Dicke-Jordan theory of gravity; see examples in Refs. [4,28,29,32-38]. In such a setup, it may still be possible to perform a scale-invariant regularization and then examine such a scale-invariant theory at the quantum level.

\section{SM WITH A SCALE-INVARIANT ONE-LOOP POTENTIAL}

\section{A. Tree-level scale-invariant potential}

Consider the SM Lagrangian with tree-level Higgs mass $m_{\phi}=0$, so it is scale invariant. The Higgs sector is weakly coupled to the "hidden" sector of the dilaton $\sigma$ with

$$
\mathcal{L}=\left|D_{\mu} H\right|^{2}+\frac{1}{2}\left(\partial_{\mu} \sigma\right)^{2}-V_{0},
$$

where

$$
H=\left(\begin{array}{c}
G^{+} \\
\frac{1}{\sqrt{2}}\left(\phi+i G^{0}\right)
\end{array}\right)
$$

and

$$
\begin{aligned}
V_{0}= & \frac{\lambda_{\phi}}{3 !}\left(H^{\dagger} H\right)^{2}+\frac{\lambda_{m}}{2}\left(H^{\dagger} H\right) \sigma^{2}+\frac{\lambda_{\sigma}}{4 !} \sigma^{4} \\
& +\frac{4 \lambda_{6}}{3} \frac{\left(H^{\dagger} H\right)^{3}}{\sigma^{2}}+\cdots
\end{aligned}
$$

where the dots stand for higher powers of $H^{\dagger} H$. The neutral Higgs $(\phi)$ and dilaton part is

$$
V(\phi, \sigma)=\frac{1}{4 !} \lambda_{\phi} \phi^{4}+\frac{1}{4} \lambda_{m} \phi^{2} \sigma^{2}+\frac{1}{4 !} \lambda_{\sigma} \sigma^{4}+\frac{\lambda_{6}}{6} \frac{\phi^{6}}{\sigma^{2}}+\cdots .
$$

We take $\lambda_{\phi}, \lambda_{\sigma}>0$ and $\lambda_{m}<0$ and that the two sectors of $\phi$ and $\sigma$ are weakly coupled, with $\left|\lambda_{m}\right|<\lambda_{\phi}$. Regarding the terms suppressed by powers of $\sigma$, they respect the (classical) scale symmetry of the action, so they can be present in the theory. They are well defined ${ }^{8}$ since $\sigma$ acquires spontaneously a vev $\langle\sigma\rangle \neq 0$ under conditions that we identify shortly [see ( $a$ ) in Eqs. (9) and (11) below]. One can expand such terms about the ground state, into an infinite sum of familiar polynomial (effective) operators:

$\begin{aligned} \lambda_{6} \frac{\phi^{6}}{\sigma^{2}} & =\lambda_{6} \frac{\phi^{6}}{\langle\sigma\rangle^{2}}\left(1-2 \frac{\sigma^{\prime}}{\langle\sigma\rangle}+3 \frac{\sigma^{\prime 2}}{\langle\sigma\rangle^{2}}+\cdots\right), \\ \sigma & =\langle\sigma\rangle+\sigma^{\prime}, \quad \sigma^{\prime} \text { : fluctuation. }\end{aligned}$

However, we prefer to use the form in Eq. (7) since it keeps manifest the scale symmetry of $\mathcal{L}$. Finally, we keep $\lambda_{6} \neq 0$ but set to 0 the coefficients of $\left(H^{\dagger} H\right)^{4} / \sigma^{4}$ and higher terms.

Consider first $\lambda_{6}=0$. We demanded spontaneous breaking of scale symmetry, so we seek the condition for which $\langle\sigma\rangle \neq 0$. The minimum of $V$ exists if derivatives $V_{\phi}=V_{\sigma}=0$, giving

$$
\begin{aligned}
& (a): \lambda_{\sigma}=\frac{9 \lambda_{m}^{2}}{\lambda_{\phi}}[1+\text { loops }] \text { and } \\
& (b): \frac{\langle\phi\rangle^{2}}{\langle\sigma\rangle^{2}}=\frac{-3 \lambda_{m}}{\lambda_{\phi}}[1+\text { loops }]
\end{aligned}
$$

so also $\langle\phi\rangle \neq 0$; here, "loops" stands for loop corrections.

Let us then assume that $\lambda_{\sigma}$ is indeed that of $(a)$ up to 9 loop effects that one can identify order by order in perturbation theory and that we ignore for the classical

\footnotetext{
${ }^{8}$ Even if we set $\lambda_{6,8, \ldots}=0$ at the electroweak scale, such terms are generated in a quantum scale-invariant theory at one loop (with a finite coefficient) [8] or as two-loop counterterms [10], so their presence is inevitable. If instead $\mu=$ constant (explicit breaking) and $\lambda_{6,8, \ldots}=0$, such terms are never generated at quantum level.

${ }^{9}$ It is actually the generalization of $(a)$ for $\lambda_{6} \neq 0$ that we shall assume to be true, see Eq. (11).
} 
discussion here. If (a) is true, we have spontaneous breaking of scale symmetry and

$$
V=\frac{1}{4 !} \lambda_{\phi} \sigma^{4}\left(\frac{\phi^{2}}{\sigma^{2}}+\frac{3 \lambda_{m}}{\lambda_{\phi}}\right)^{2}
$$

with $V=0$ at the minimum. A flat direction, corresponding to the Goldstone of scale symmetry (dilaton), exists in the plane $(\phi, \sigma)$. The neutral Higgs acquires a mass $m_{\tilde{\phi}}^{2}=$ $\left(\lambda_{\phi} / 3\right)\left(1-3 \lambda_{m} / \lambda_{\phi}\right)\langle\phi\rangle^{2}$, while the electroweark (EW) Goldstone bosons are massless. Thus, spontaneous scale symmetry breaking triggers EW symmetry breaking, with a vacuum energy $V=0$.

Consider now $\lambda_{6} \neq 0$, with $\lambda_{6}>0$ for a well-defined $V$ at large $\phi$. Then, Eqs. (9) become ${ }^{10}$

$(a): \lambda_{\sigma}=\rho_{0}^{2}\left[2 \lambda_{6} \rho_{0}^{4}-3 \lambda_{m}\right]+$ loops, where

$(b): \rho_{0}^{2} \equiv \frac{\langle\phi\rangle^{2}}{\langle\sigma\rangle^{2}}=\frac{1}{12 \lambda_{6}}\left[-\lambda_{\phi}+\left(\lambda_{\phi}^{2}-72 \lambda_{6} \lambda_{m}\right)^{1 / 2}\right]+$ loops.

We assume from now on that $\lambda_{\sigma}$ is indeed given by relation $(a)$, up to small quantum corrections (ignored here), to ensure spontaneous scale symmetry breaking; this relation is "protected" by scale symmetry. The potential is then

$$
V=\frac{\lambda_{6}}{6} \sigma^{4}\left(\frac{\phi^{2}}{\sigma^{2}}-\rho_{0}^{2}\right)^{2}\left(\frac{\phi^{2}}{\sigma^{2}}+\xi_{0}^{2}\right)
$$

in agreement with (3). Here, $\xi_{0}^{2}=\left(\lambda_{\phi}+\right.$ $\left.2\left(\lambda_{\phi}^{2}-72 \lambda_{6} \lambda_{m}\right)^{1 / 2}\right) /\left(12 \lambda_{6}\right)>0$. If $\lambda_{6} \rightarrow 0$, one recovers Eq. (10). ${ }^{11}$ The neutral Higgs mass can again be computed and recovers the above value for small ${ }^{12} \lambda_{6}$; the dilaton is again massless, with the flat direction mildly changed by $\lambda_{6}$. To conclude, spontaneous scale symmetry breaking triggers EW symmetry breaking and ensures $V=0$ on the ground state. We would like to know if this can remain true at quantum level.

The scale $\langle\sigma\rangle$ of new physics beyond SM should be larger than $\langle\phi\rangle \sim \mathcal{O}(100) \mathrm{GeV}$. In the Brans-Dicke-Jordan theory of gravity (not considered here) that can generalize this study, one actually expects $\langle\sigma\rangle \sim M_{\text {Planck. So, a }}$ hierarchy $\langle\phi\rangle \ll\langle\sigma\rangle$ may be generated dynamically $[28,29]$. Here, we take a common view of a very weak coupling of the hidden $(\sigma)$ to visible $(\phi)$ sector: $\left|\lambda_{m}\right| \ll \lambda_{\phi}$

\footnotetext{
${ }^{10}$ Equations (11) for small $\lambda_{6}$ become $\lambda_{\sigma}=\left(9 \lambda_{m}^{2} / \lambda_{\phi}\right)[1+$ $\mathcal{O}\left(\lambda_{6}\right)+$ loops $]$ and $\rho_{0}^{2}=\left(-3 \lambda_{m} / \lambda_{\phi}\right)\left[1+\mathcal{O}\left(\lambda_{6}\right)+\right.$ loops $]$.

${ }^{11}$ With $y=\phi^{2} / \sigma^{2}, V=\left(\lambda_{\phi} \sigma^{4} / 4 !\right)\left(y+3 \lambda_{m} / \lambda_{\phi}\right)\left[y+3 \lambda_{m} / \lambda_{\phi}+\right.$ $\left.\left(4 \lambda_{6} / \lambda_{\phi}\right)\left(y^{2}-3 y \lambda_{m} / \lambda_{\phi}+9 \lambda_{m}^{2} / \lambda_{\phi}^{2}\right)\right]+\mathcal{O}\left(\lambda_{6}\right)$.

${ }^{12}$ One has $m_{\tilde{\phi}}^{2}=\left(-2+\frac{\lambda_{\phi}}{6 \lambda_{6}}\right) \lambda_{m}\langle\sigma\rangle^{2}+\rho_{0}^{2}\left[\frac{\lambda_{\phi}}{3}\left(\frac{\lambda_{\phi}}{6 \lambda_{6}}-1\right)-2 \lambda_{m}\right]\langle\sigma\rangle^{2}=$ $-\lambda_{m}\left(1-\frac{3 \lambda_{m}}{\lambda_{\phi}}\right)\langle\sigma\rangle^{2}+\mathcal{O}\left(\lambda_{6}\right)$.
}

[31]; then, ${ }^{13}$ from Eq. (11), $\lambda_{\sigma} \ll\left|\lambda_{m}\right|$. This classical "tuning" can ensure a hierarchy of scales $\langle\phi\rangle \ll\langle\sigma\rangle\left(\lambda_{6}\right.$ only brings subleading corrections, since the hierarchy is controlled by $\lambda_{m}$, the main coupling of the two sectors). Note that this is not a tuning in the sense of the cancellation of mass scales, seen in the mass hierarchy problem.

This concludes the picture of the classical potential with scale symmetry. At the quantum level, one question is whether the (quantum) scale symmetry, when spontaneously broken, maintains the hierarchy $m_{\tilde{\phi}}^{2} \sim\langle\phi\rangle^{2} \ll\langle\sigma\rangle^{2}$ without additional tuning of the couplings. If quantum corrections $\lambda_{\phi}^{2}\langle\sigma\rangle^{2}$ are generated, a tuning of the Higgs selfcoupling $\lambda_{\phi}$ would be needed, and this would reintroduce the hierarchy problem.

\section{B. One-loop scale-invariant potential}

Let us compute the one-loop potential by preserving scale symmetry at quantum level and thus avoid its explicit breaking by the UV regularization. The method is described in Refs. [4,6-10]. To do this, note that we already have a vev $\langle\sigma\rangle$ that can act as subtraction scale. The starting point is in $d=4-2 \epsilon$ dimensions where the treelevel potential is modified into

$$
\tilde{V}=\mu(\sigma)^{2 \epsilon} V, \quad \mu(\sigma)=z \sigma^{1 /(1-\epsilon)} .
$$

$\tilde{V}$ is thus scale invariant in $d=4-2 \epsilon$. The function $\mu(\sigma)$ generates a subtraction scale $\mu(\langle\sigma\rangle)$ when $\sigma$ acquires a vev spontaneously. The definition of $\mu(\sigma)$ follows on dimensional grounds, with $z$ an arbitrary dimensionless subtraction parameter [7]. If we set $\mu(\sigma)=$ constant, one immediately recovers the traditional DR scheme that explicitly breaks the scale symmetry in $d=4-2 \epsilon$. We thus have two possible analytical continuations to $d=4-2 \epsilon$ of the classical scale-invariant theory in $d=4$ : one is scale invariant [Eq. (13)], and the other is not ( $\mu=$ constant), and they lead to distinct quantum theories (of different symmetry) $[8,10]$, as discussed below. The one-loop potential in $d=4-2 \epsilon$ is then $[8,10]^{14}$

$$
V_{1}=\tilde{V}-\frac{i}{2} \int \frac{d^{d} p}{(2 \pi)^{d}} \operatorname{Tr} \ln \left[p^{2}-\tilde{V}_{i j}+i \varepsilon\right] .
$$

This is computed in the Landau gauge. The fielddependent squared masses are eigenvalues of the matrix of second derivatives denoted ${ }^{15} \tilde{V}_{i j}$, where subscripts $i, j$

\footnotetext{
${ }^{13}$ This hierarchy is stable under the renormalization group [31] due to a shift symmetry, $\sigma \rightarrow \sigma+$ constant.

${ }^{14} V_{1}$ is derived in $d=4-2 \epsilon$ via usual diagrammatic or functional methods in effective theories and remains valid in the presence of the $\lambda_{6} \phi^{6} / \sigma^{2}$ term, which is just a sum of familiar polynomial operators; see Eq. (8).

${ }^{15} \tilde{V}_{i j}=\mu^{2 \epsilon}\left[V_{i j}+2 \epsilon \mu^{-2} N_{i j}\right]+\mathcal{O}\left(\epsilon^{2}\right) ; \quad N_{i j} \equiv \mu\left\{\frac{\partial \mu}{\partial s_{i}} \frac{\partial V}{\partial s_{j}}+\frac{\partial \mu}{\partial s_{j}} \frac{\partial V}{\partial s_{i}}\right\}+$ $\left\{\mu \frac{\partial^{2} \mu}{\partial s_{i} \partial s_{j}}-\frac{\partial \mu}{\partial s_{i}} \frac{\partial \mu}{\partial s_{j}}\right\} V$.
} 
stand for the EW Goldstone scalars $G^{0}, \operatorname{Re}\left(G^{+}\right), \operatorname{Im}\left(G^{+}\right)$, neutral Higgs $\phi$, and dilaton $\sigma$. Unlike the EW Goldstone modes or fermions and gauge bosons, the field-dependent masses of $\phi$ and $\sigma$ acquire a correction $\propto \epsilon$ relative to their values induced by $V$ alone, from derivatives of $\mu(\sigma)$,

$m_{t}^{2}=\frac{\mu(\sigma)^{2 \epsilon}}{2} h_{t}^{2} \phi^{2}, \quad m_{W}^{2}=\frac{\mu(\sigma)^{2 \epsilon}}{4} g_{2}^{2} \phi^{2}$,

$m_{Z}^{2}=\frac{\mu(\sigma)^{2 \epsilon}}{4}\left(g_{1}^{2}+g_{2}^{2}\right) \phi^{2}$,

$m_{G}^{2}=\frac{\mu(\sigma)^{2 \epsilon}}{6}\left[\lambda_{\phi} \phi^{2}+3 \lambda_{m} \sigma^{2}+6 \lambda_{6} \frac{\phi^{4}}{\sigma^{2}}\right]$,

$M_{k}^{2}=\mu(\sigma)^{2 \epsilon}\left[m_{k}^{2}+\epsilon \delta_{k}\right], \quad k=\phi, \sigma$,

where $m_{t}\left(h_{t}\right)$ is the field-dependent top mass (Yukawa coupling), $m_{W, Z}$ denote the gauge boson masses, and $m_{G}$ denote the three EW Goldstone field-dependent masses. Finally, $M_{k}^{2}$ are eigenvalues of $\tilde{V}_{\alpha \beta}$, while $m_{k}^{2}$ are eigenvalues of the $2 \times 2$ submatrix $V_{\alpha \beta}$ of $V_{i j}$ with $^{16} V_{\alpha \beta}=\partial^{2} V / \partial \alpha \partial \beta$, $\alpha, \beta=\phi, \sigma$. Then, one finds at one loop $\left[\kappa=(4 \pi)^{2}\right]$

$$
\begin{aligned}
V_{1}= & \mu(\sigma)^{2 \epsilon}\left\{V-\frac{1}{4 \kappa}\left[\sum_{j=\phi, \sigma ; G, W, Z, t} n_{j} m_{j}^{4}(\phi, \sigma)\right.\right. \\
& \left.\left.\times\left(\frac{1}{\epsilon}-\ln \frac{m_{j}^{2}(\phi, \sigma)}{c_{j} \mu^{2}(\sigma)}\right)+\frac{4\left(V_{\alpha \beta} N_{\beta \alpha}\right)}{\mu^{2}(\sigma)}\right]\right\},
\end{aligned}
$$

with summation over $\alpha, \beta=\phi, \sigma$ and $N_{\alpha \beta}=\mu\left(\mu_{\alpha} V_{\beta}+\right.$ $\left.\left.\mu_{\beta} V_{\alpha}\right)-\mu_{\alpha} \mu_{\beta}\right) V$ and $\mu_{\alpha}=\partial \mu / \partial \alpha$. Also, $n_{j}=$ $\{3,1,6,3,-12\}$ for $j=\{G, S, W, Z, t\}$, with $S=\phi, \sigma$; $c_{j}=4 \pi e^{3 / 2-\gamma_{E}}$ if $j=\phi, \sigma, t, G$, and $c_{j}=4 \pi e^{5 / 6-\gamma_{E}}$ if $j=W, Z$. The one-loop term $\left(V_{\alpha \beta} N_{\beta \alpha}\right)$ is a new correction, absent in the case of $\mu=$ constant (i.e., explicit scale symmetry breaking by the regularization).

The poles in the one-loop Lagrangian are cancelled by the counterterm $\delta L_{1},{ }^{17}$

$$
\begin{aligned}
\delta L_{1} \equiv & \frac{1}{2}\left(Z_{\phi}-1\right)\left(\partial_{\mu} \phi\right)^{2}+\frac{1}{2}\left(Z_{\sigma}-1\right)\left(\partial_{\mu} \phi\right)^{2} \\
& -\mu(\sigma)^{2 \epsilon}\left\{\frac{1}{4 !}\left(Z_{\lambda_{\phi}}-1\right) \lambda_{\phi} \phi^{4}+\frac{1}{4}\left(Z_{\lambda_{m}}-1\right) \lambda_{m} \phi^{2} \sigma^{2}\right. \\
& \left.\left.+\frac{1}{4 !}\left(Z_{\lambda_{\sigma}}-1\right) \lambda_{\sigma} \sigma^{4}\right)+\sum_{j=3,4,5,6} \frac{1}{2 j}\left(Z_{\lambda_{2 j}}-1\right) \lambda_{2 j} \frac{\phi^{2 j}}{\sigma^{2 j-4}}\right\} .
\end{aligned}
$$

\footnotetext{
${ }^{16}$ In general, in terms of derivatives of tree-level $\mathrm{V}$, $m_{k}^{2}=\frac{1}{2}\left[\operatorname{Tr}\left(V_{\alpha \beta}\right) \pm\left[\left(\operatorname{Tr} V_{\alpha \beta}\right)^{2}-4 \operatorname{det} V_{\alpha \beta}\right]^{1 / 2}\right] \quad$ and also $\delta_{k}=\mu(\sigma)^{-2}\left\{\operatorname{Tr}\left(N_{\alpha \beta}\right) \pm\left[\left(\operatorname{Tr} V_{\alpha \beta}\right)\left(\operatorname{Tr} N_{\alpha \beta}\right)-2 \rho\right] /\left[\left(\operatorname{Tr} V_{\alpha \beta}\right)^{2}-\right.\right.$ $\left.\left.4 \operatorname{det} V_{\alpha \beta}\right]^{1 / 2}\right\}$. The expression of $\rho$ is $\rho=V_{\phi \phi} N_{\sigma \sigma}+V_{\sigma \sigma} N_{\phi \phi}-$ $2 V_{\phi \sigma} N_{\phi \sigma}$, where $N_{\phi \phi}=0, N_{\sigma \sigma}=z^{2}\left(2 \sigma V_{\sigma}-V\right), N_{\phi \sigma}=z^{2} \sigma V_{\phi}$.

${ }^{17}$ One can use $\sum_{k=\phi, \sigma} m_{k}^{4}=V_{\phi \phi}^{2}+V_{\sigma \sigma}^{2}+2 V_{\phi \sigma}^{2}$.
}

Introducing the notation

$$
Z_{\xi}=1+\frac{1}{\epsilon} \frac{\gamma_{\xi}}{\kappa}, \quad \xi=\lambda_{\phi}, \lambda_{\phi}, \text { etc },
$$

one identifies

$\gamma_{\lambda_{\phi}}=\frac{3}{2 \lambda_{\phi}}\left(\frac{3}{2} g_{2}^{4}+\frac{3}{4}\left(g_{1}^{2}+g_{2}^{2}\right)^{2}-12 h_{t}^{4}+\frac{4}{3} \lambda_{\phi}^{2}+\lambda_{m}^{2}+32 \lambda_{m} \lambda_{6}\right)$,

$\gamma_{\lambda_{m}}=\frac{1}{2}\left(2 \lambda_{\phi}+\lambda_{\sigma}+4 \lambda_{m}\right)$,

$\gamma_{\lambda_{\sigma}}=\frac{3}{2}\left(\lambda_{\sigma}+4 \lambda_{m}^{2} / \lambda_{\sigma}\right)$.

Notice that $\lambda_{6}$ contributes to $\gamma_{\lambda_{\phi}}$ and to the beta function of $\lambda_{\phi}$ (see later in the paper, Eq. (26)). Finally,

$\gamma_{\lambda_{6}}=\frac{3}{2}\left(6 \lambda_{\phi}-8 \lambda_{m}+\lambda_{\sigma}\right), \quad \gamma_{\lambda_{8}}=\frac{2 \lambda_{6}}{\lambda_{8}}\left(28 \lambda_{6}+\lambda_{m}\right)$,

$\gamma_{\lambda_{10}}=20 \frac{\lambda_{6}^{2}}{\lambda_{10}}, \quad \gamma_{\lambda_{12}}=\frac{3 \lambda_{6}^{2}}{\lambda_{12}}$.

Therefore, the nonpolynomial operator $\lambda_{6} \phi^{6} / \sigma^{2}$ in the tree-level $V$ generated new nonpolynomial counterterms up to and including $\phi^{12} / \sigma^{8}$, of couplings $\propto \lambda_{6}$. This effect is independent of whether the quantum calculation respects the scale symmetry or not (i.e., $\mu \sim \sigma$ or $\mu=$ constant). The generalization to more such operators at the tree level is immediate.

The SM one-loop potential $U_{1}$ is then

$$
U_{1}=V+V^{(1)}+V^{(1, n)},
$$

where

$$
\begin{aligned}
V^{(1)}= & \frac{1}{4 \kappa} \sum_{j=\phi, \sigma ; G, t, W, Z} n_{j} m_{j}^{4}(\phi, \sigma) \ln \frac{m_{j}^{2}(\phi, \sigma)}{c_{j}(z \sigma)^{2}}, \\
V^{(1, n)}= & \frac{1}{48 \kappa}\left[\left(-16 \lambda_{m} \lambda_{\phi}-18 \lambda_{m}^{2}+\lambda_{\phi} \lambda_{\sigma}\right) \phi^{4}\right. \\
& -\lambda_{m}\left(48 \lambda_{m}+25 \lambda_{\sigma}\right) \phi^{2} \sigma^{2}-7 \lambda_{\sigma}^{2} \sigma^{4} \\
& +\left(\lambda_{\phi} \lambda_{m}+6 \lambda_{6} \lambda_{\sigma}\right) \frac{\phi^{6}}{\sigma^{2}}+8 \lambda_{6}\left(4 \lambda_{\phi}-2 \lambda_{m}\right) \frac{\phi^{8}}{\sigma^{4}} \\
& \left.+\lambda_{6}\left(192 \lambda_{6}+2 \lambda_{\phi}\right) \frac{\phi^{10}}{\sigma^{6}}+40 \lambda_{6}^{2} \frac{\phi^{12}}{\sigma^{8}}\right] .
\end{aligned}
$$

$U_{1}$ is manifestly scale invariant. First, the ColemanWeinberg term is modified into a scale-invariant form $V^{(1)}$, where we finally replaced $\mu(\sigma)=z \sigma$ [see Eq. (13) for $\epsilon \rightarrow 0]$. Note that $V^{(1)}$ contains new terms of the form $\phi^{8} / \sigma^{4} \ln [\ldots], \phi^{6} / \sigma^{2} \ln [\ldots]$ of coefficients $\propto \lambda_{6}$, that 
originate from $m_{G}^{4}(\phi, \sigma)$. In the usual DR scheme, $V^{(1)}$ has the same form, with $(z \sigma) \rightarrow \mu$.

There is also a finite one-loop contribution $V^{(1, n)}$ due to "evanescent" corrections $(\alpha \epsilon)$ to the field-dependent masses of $\phi$ and $\sigma$ [Eq. (15)], induced by derivatives of $\mu \sim \sigma$. Therefore, $V^{(1, n)}$ is not present in the other case of $\mu=$ constant when the regularization breaks the scale symmetry; thus, $V^{(1, n)}$ can distinguish between these two cases at one loop. Further, in the classical decoupling limit of the hidden sector from the SM, $\lambda_{m} \rightarrow 0$ and $\lambda_{6} \rightarrow 0$, and then $V^{(1, n)}$ vanishes. $V^{(1, n)}$ also contains terms nonpolynomial in fields like $\lambda_{m} \lambda_{\phi} \phi^{6} / \sigma^{2}$ that remain present even if we set $\lambda_{6}=0 .{ }^{18}$ At two loops, such nonpolynomial operators, including higher-order $\phi^{8} / \sigma^{4}$, etc., emerge as two-loop counterterms [10] even if we set $\lambda_{6}=0 .{ }^{19}$

Although we do not show it, one can immediately Taylor expand both $V^{(1)}$ and $V^{(1, n)}$ about the nonzero vev of $\sigma$, with $\sigma=\langle\sigma\rangle+\sigma^{\prime}$ (and eventually of $\phi$, too, $\phi=\langle\phi\rangle+\phi^{\prime}$ ). One then obtains a representation that contains an infinite sum of polynomial operators in the field fluctuations $\left(\phi^{\prime}, \sigma^{\prime}\right)$ suppressed by powers of $\langle\sigma\rangle$. However, in this case, manifest scale symmetry of the quantum result is lost.

\section{One-loop beta functions and Callan-Symanzik equation}

To check the quantum consistency of the scalar potential, we verify the Callan-Symanzik equation for it. This is to ensure that the physics is independent of the subtraction scale $\mu(\langle\sigma\rangle)=z\langle\sigma\rangle$. To this purpose, we need the one-loop beta functions of all couplings, including those of the nonpolynomial operators. These are computed from the condition that the bare coupling is independent of the subtraction parameter ${ }^{20} z$. For example, $d /(d \ln z) \lambda_{\phi}^{B}=0$, where $\lambda_{\phi}^{B}=\mu(\sigma)^{2 \epsilon} \lambda_{\phi} Z_{\phi}^{-2} Z_{\lambda_{\phi}}$ and $\phi_{B}^{2}=Z_{\phi} \phi^{2}$. Using these relations, the beta function that is $\beta_{\lambda_{\phi}}=d \lambda_{\phi} / d(\ln z)$ becomes

$$
\beta_{\lambda_{\phi}}=-2 \epsilon \lambda_{\phi}+\frac{2 \lambda_{\phi}}{\kappa} \alpha_{j} \frac{d}{d \alpha_{j}}\left[\gamma_{\lambda_{\phi}}-2 \gamma_{\phi}\right]
$$

with summation over $j$ with $\alpha_{j}=g_{1}^{2}, g_{2}^{2}, h_{t}^{2}, \lambda_{\phi}, \lambda_{m}, \lambda_{\sigma}, \lambda_{6}$, $\lambda_{8}$, etc. Next, using notation (18), one has

\footnotetext{
${ }^{18}$ Assuming one set $\lambda_{6}=0$ at tree level, some other subtraction scheme could eventually remove finite $\phi^{4}, \phi^{2} \sigma^{2}$, or $\sigma^{4}$ terms in $V^{(1, n)}$ but could not remove the remaining $\lambda_{m} \lambda_{\phi} \phi^{6} / \sigma^{2}$ that does not vanish for $\lambda_{6}=0$.

${ }^{19}$ The two-loop beta functions of such terms are nonzero even if $\lambda_{6}=0$, so setting these to zero (at some scale) will not remove them since they are again generated at a different scale [10].

${ }^{20}$ The dimensionless parameter $z$ tracks the dependence on the subtraction scale $\mu(\langle\sigma\rangle)=z\langle\sigma\rangle$.
}

$$
\gamma_{\phi}=\frac{1}{\kappa}\left(\frac{3}{4} g_{1}^{2}+\frac{9}{4} g_{2}^{2}-3 h_{t}^{2}\right), \quad \gamma_{\sigma}=0,
$$

which can easily be computed in a scale-invariant way. ${ }^{21}$ Relations similar to Eq. (24) exist for the other beta functions. We then find

$$
\begin{aligned}
& \beta_{\lambda_{\phi}}=\frac{2 \lambda_{\phi}}{\kappa}\left(\gamma_{\lambda_{\phi}}-2 \gamma_{\phi}\right), \\
& \beta_{\lambda_{m}}=\frac{2 \lambda_{m}}{\kappa}\left(\gamma_{\lambda_{m}}-\gamma_{\phi}\right), \\
& \beta_{\lambda_{\sigma}}=\frac{2 \lambda_{\sigma}}{\kappa} \gamma_{\lambda_{\sigma}} .
\end{aligned}
$$

$\beta_{\lambda_{\phi}}$ includes a correction due to $\lambda_{6}$, which is the coupling of the nonpolynomial term that we included in the classical potential Eq. (6). These one-loop beta functions are identical to those of the similar theory with a regularization that breaks scale symmetry explicitly $(\mu=$ constant $) .{ }^{22} \mathrm{We}$ find in a similar way

$\beta_{\lambda_{6}}=\frac{2 \lambda_{6}}{\kappa}\left(\gamma_{\lambda_{6}}-3 \gamma_{\phi}\right), \quad \beta_{\lambda_{8}}=\frac{2 \lambda_{8}}{\kappa}\left(\gamma_{\lambda_{8}}-4 \gamma_{\phi}\right)$,

$\beta_{\lambda_{10}}=\frac{2 \lambda_{10}}{\kappa}\left(\gamma_{\lambda_{10}}-5 \gamma_{\phi}\right), \quad \beta_{\lambda_{12}}=\frac{2 \lambda_{12}}{\kappa}\left(\gamma_{\lambda_{12}}-6 \gamma_{\phi}\right)$.

These beta functions of the nonpolynomial operators are difficult to obtain by other methods (diagrammatic, etc.). This justifies keeping these operators in a scale symmetric form [Eq. (7)], rather than expanding them about the ground state in a series of polynomial operators [Eq. (8)].

The Callan-Symanzik equation of the scalar potential states the independence of the potential of the subtraction scale. At one loop, this gives [7]

$$
\begin{aligned}
\frac{d}{d \ln z} U_{1}\left(\phi, \sigma, \alpha_{k}\right) & =\left(z \frac{\partial}{\partial z}+\beta_{\alpha_{k}} \frac{\partial}{\partial \alpha_{k}}+\gamma_{\phi} \phi \frac{\partial}{\partial \phi}\right) U_{1}\left(\phi, \sigma, \alpha_{k}\right) \\
& =\mathcal{O}\left(\alpha_{j}^{3}\right) .
\end{aligned}
$$

Here, $\alpha_{k}$ denote the couplings $\lambda_{\phi}, \lambda_{\sigma}, \lambda_{m}, g_{1}^{2}, g_{2}^{2}, h_{t}^{2}, \lambda_{6}, \ldots \lambda_{12}$, which were found to have nonzero beta functions. Further, $\gamma_{\phi}=\frac{\partial \ln \phi}{\partial \ln z}=-\frac{1}{2} \frac{\partial \ln Z_{\phi}}{\partial \ln z}$ was found in Eqs. (25) and (18), while $\gamma_{\sigma}=0$. Finally, $U_{1}\left(\phi, \sigma, \alpha_{k}\right)$ denotes the potential found in Eq. (21) with the observation that all couplings are

\footnotetext{
${ }^{21} \gamma_{\phi}$ and $\gamma_{\sigma}$ have the same expression as when $\mu=$ constant scale.

${ }^{22}$ However, at two-loop order, the beta functions start to differ [10] in our case of spontaneous scale symmetry breaking from the case of explicit breaking (by the regularization with $\mu=$ constant). In this case, the evanescent corrections $\alpha \epsilon$ to scalar field-dependent masses (Higgs and dilaton) of the potential "meet" the $1 / \epsilon^{2}$ usual two-loop poles, to bring new poles $\epsilon \times 1 / \epsilon^{2} \sim 1 / \epsilon$ that demand new counterterms, thus modifying the beta functions; see Ref. [10] for details.
} 
now replaced by their "running" versions. In particular, the tree-level potential (part of $U_{1}$ ) is supplemented with the following terms with running couplings $\lambda_{8,10,12}$ :

$$
V \rightarrow V+\frac{\lambda_{8}}{8} \frac{\phi^{8}}{\sigma^{2}}+\frac{\lambda_{10}}{10} \frac{\phi^{10}}{\sigma^{6}}+\frac{\lambda_{12}}{12} \frac{\phi^{12}}{\sigma^{8}} .
$$

These terms are present since the couplings $\lambda_{8,10,12}$ (which had boundary values set to 0 at the $\mathrm{EW}$ scale, unlike $\lambda_{6} \neq 0$ ), have nonzero beta functions.

The only explicit $z$-dependent part in $U_{1}$ comes through the Coleman-Weinberg part $V^{(1)}$ of Eq. (21), while the terms involving the beta functions and anomalous dimension act only on the tree-level part of the potential, in our one-loop approximation.

With the above results, checking the Callan-Symanzik equation is immediate. We stress that this is verified in the presence of the nonpolynomial operators that actually correspond to infinitely many polynomial operators when expanded about the ground state.

In conclusion, the potential is indeed independent of the subtraction scale $z\langle\sigma\rangle$, so one can take any value for it. It is customary to set the subtraction scale equal to $\langle\phi\rangle$, to minimize the $\log$ terms in the potential. In our scaleinvariant approach, $\mu(\sigma)=z \sigma$, so after scale symmetry breaking, $\mu(\langle\sigma\rangle)=\langle\phi\rangle$ if we take $z=\langle\phi\rangle /\langle\sigma\rangle$, and we do so below. This means the couplings and fields are evaluated at the scale $\langle\phi\rangle$.

\section{One-loop Higgs mass}

The one-loop corrected potential is scale invariant, and it has a flat direction, ${ }^{23}$ the dilaton, which remains massless at the quantum level (unlike in the traditional DR scheme of $\mu=$ constant). We can compute the Higgs mass $m_{\tilde{\phi}}$ at one loop by using

$$
m_{\tilde{\phi}}^{2}=\left(U_{1}\right)_{\sigma \sigma}+\left.\left(U_{1}\right)_{\phi \phi}\right|_{\min },
$$

where the subscripts denote derivatives with respect to the fields shown. We calculate the new ground state and the correction $\delta m_{\tilde{\phi}}^{2}$ to classical $m_{\tilde{\phi}}^{2}$ in the limit of an ultraweak coupling of the visible to the hidden sector $\left|\lambda_{m}\right| \ll \lambda_{\phi}$ (giving $\lambda_{\sigma} \ll\left|\lambda_{m}\right|$ ). This was motivated earlier in that it ensures a classical hierarchy $\langle\phi\rangle \ll\langle\sigma\rangle$. The new ground state is modified to

$\frac{\langle\phi\rangle^{2}}{\langle\sigma\rangle^{2}}=\frac{-3 \lambda_{m}}{\lambda_{\phi}}[1+\zeta], \quad \zeta=-\frac{\lambda_{\phi}}{4 \kappa}\left[4 \ln \left(\lambda_{\phi} / 2\right)-8\right]$.

With the notation $g^{2} \equiv g_{1}^{2}+g_{2}^{2}$, the one-loop correction is

\footnotetext{
${ }^{23}$ See the previous discussion in the Introduction around Eqs. (2).
}

$$
\begin{aligned}
\delta m_{\tilde{\phi}}^{2}= & \frac{-\lambda_{m}}{\lambda_{\phi}} \frac{\langle\sigma\rangle^{2}}{16 \kappa}\left\{2 7 \left[g^{4}\left(\ln \frac{g^{2}}{4}+\frac{1}{3}\right)+2 g_{2}^{4}\left(\ln \frac{g_{2}^{2}}{4}+\frac{1}{3}\right)\right.\right. \\
& \left.\left.-16 h_{t}^{4}\left(\ln \frac{h_{t}^{2}}{2}-\frac{1}{3}\right)\right]+4 \lambda_{\phi}^{2}\left[5 \ln \frac{\lambda_{\phi}^{2}}{12}-8+\ln 27\right]\right\} .
\end{aligned}
$$

This quantum correction remains proportional to $\lambda_{m}\langle\sigma\rangle^{2} \sim \mathcal{O}(100 \mathrm{GeV})$, just like the tree-level value. Thus, the initial classical hierarchy $m_{\tilde{\phi}} \sim\langle\phi\rangle \ll\langle\sigma\rangle$ is stable in the presence of quantum corrections, without any quantum tuning of the couplings $\lambda_{\phi, m, \sigma}$, in agreement with previous results $[4,8] .{ }^{24,25}$ An additional correction from $\lambda_{6} \neq 0$ does not change this result since it is subleading in the limit of the ultraweak coupling considered here (being suppressed by the large $\langle\sigma\rangle$ ). Finally, the spontaneous breaking of scale symmetry used here avoids the constraint of Ref. [39] (derived using explicit breaking by the usual DR scheme) that demands new physics at the $\mathrm{TeV}$ scale.

\section{E. What about the dilatation anomaly?}

Let us analyze the situation of the dilatation current $D^{\mu}$ and its divergence [6,7]. For a set of fields $\phi_{j}(\phi, \sigma$, etc.),

$$
\begin{aligned}
D^{\mu} & =\frac{\partial \mathcal{L}}{\partial\left(\partial_{\mu} \phi_{j}\right)}\left(x^{\nu} \partial_{\nu} \phi_{j}+d_{\phi}\right)-x^{\mu} \mathcal{L}, \\
\partial_{\mu} D^{\mu} & =\left(d_{\phi}+1\right)\left(\partial_{\mu} \phi_{j}\right) \frac{\partial \mathcal{L}}{\partial\left(\partial_{\mu} \phi_{j}\right)}+d_{\phi} \phi_{j} \frac{\partial \mathcal{L}}{\partial \phi_{j}}-d \mathcal{L},
\end{aligned}
$$

with $d_{\phi}$ the mass dimension of $\phi, d_{\phi}=(d-2) / 2$ for a scalar in $d$ dimensions. For standard kinetic terms and using the equations of motion, we find for a potential $\mathcal{V}$ in $d$ dimensions

$$
\partial_{\mu} D^{\mu}=d \mathcal{V}-\frac{d-2}{2} \phi_{j} \frac{\partial \mathcal{V}}{\partial \phi_{j}}
$$

Consider now that $\mathcal{V}$ is scale invariant at both classical and quantum level as in our case, ${ }^{26}$ Eq. (13) [also Eq. (21)]. Therefore, for a dimensionless parameter $\rho, \mathcal{V}$ has the property ${ }^{27} \mathcal{V}\left(\rho \phi_{j}\right)=\rho^{2 d /(d-2)} \mathcal{V}\left(\phi_{j}\right)$ in $d=4-2 \epsilon$ dimensions (homogeneous function). Differentiating this

\footnotetext{
${ }^{24}$ In particular, there is no term $\lambda_{\phi}\langle\sigma\rangle^{2}$ that would require tuning the Higgs self-coupling $\lambda_{\phi}$, etc.

${ }^{25}$ For the physical Higgs mass, there is also the usual correction of running from $p^{2}=0$ to $p^{2}=m_{h}^{2}$.

${ }^{26} \mathrm{We}$ have $\mathcal{V}=\mu(\sigma)^{2 \epsilon} V$ in Eqs. (13), while in Eq. (21), $\mathcal{V}=\mu(\sigma)^{2 \epsilon} U_{1}$ before $\epsilon \rightarrow 0$.

${ }^{27}$ This property is shown using that $\mathcal{V}\left(\phi_{j}\right)=\phi_{k}^{\xi} \mathcal{V}\left(\phi_{j} / \phi_{k}\right)$, $k=$ fixed; since $\left[\mathcal{V}\left(\phi_{j}\right)\right]=d, \mathcal{V}\left(\phi_{j} / \phi_{k}\right]=0$ and $\left[\phi_{j}\right]=(d-2) / 2$, then $\xi=2 d /(d-2)$. Then, $\mathcal{V}\left(\rho \phi_{j}\right)=\left(\rho \phi_{k}\right)^{\xi} \mathcal{V}\left(\phi_{j} / \phi_{k}\right)=\rho^{\xi} \mathcal{V}\left(\phi_{j}\right)$ with $\xi$ as above.
} 
equation with respect to $\rho$ and then taking $\rho \rightarrow 1$ gives $2 d /(d-2) \mathcal{V}=\phi_{j} \partial \mathcal{V} / \partial \phi_{j}$, so the rhs of Eq. (34) then vanishes. Therefore, $\partial_{\mu} D^{\mu}=0$ at both the classical and quantum levels, so there is no anomalous breaking of the quantum scale symmetry. Nevertheless, the couplings still "run" and have nonzero beta functions [Eq. (26)] with their corresponding poles in $\mathcal{L}$.

To understand this better, let us also see what happens if $\mathcal{V}$ is not scale invariant in $d=4-2 \epsilon$. This happens when $\mathcal{V}=\mu^{2 \epsilon} V\left(\phi_{j}\right)$, which is the case of the traditional DR scheme with explicit scale symmetry breaking, with $\mu$ a fixed scale (not a function of the fields) and $V$ the potential, scale invariant in $d=4$ (assuming no mass terms). Then, $V\left(\rho \phi_{j}\right)=\rho^{4} V\left(\phi_{j}\right)$, but $\mathcal{V}$ is no longer scale invariant in $d=4-2 \epsilon$. Then, from Eq. (34),

$\partial_{\mu} D^{\mu}=d \mu^{2 \epsilon} V-2(d-2) \mu^{2 \epsilon} V\left(\phi_{j}\right)=2 \epsilon \mu^{2 \epsilon} V=2 \epsilon \mu^{2 \epsilon} \lambda_{j} \frac{\partial V}{\partial \lambda_{j}}$.

While at the classical level the rhs vanishes when $\epsilon \rightarrow 0$, at the quantum level, the quartic couplings $\lambda_{j}$ in $V$ acquire a pole $\beta_{\lambda_{j}} / \epsilon$, which cancels the $\epsilon$ in front, to give a finite nonzero rhs $\partial_{\mu} D^{\mu} \propto \beta_{\lambda_{j}}\left(\partial \mathcal{L} / \partial \lambda_{j}\right)$. This is the familiar scale anomaly breaking of the conservation of this current in the traditional DR scheme. ${ }^{28,29}$

In conclusion, it is scale invariance of the action in $d=4-2 \epsilon$ that ensures that no scale anomaly is present. This invariance in $d=4-2 \epsilon$ is lost in the usual DR regularization with explicit breaking ( $\mu=$ constant). Thus, the vanishing of the beta function is not a necessary condition for the theory to be scale invariant; one must also specify how the quantum theory was regularized, with or without respecting its scale symmetry. In other words, the nonvanishing of the beta function does not mean the theory cannot be scale invariant.

\section{F. Further remarks}

As mentioned, the vacuum energy vanishes in models with scale symmetry or with spontaneous breaking of it; see the discussion after Eq. (2). This protection remains in place at the quantum level, provided this symmetry is respected by the quantum calculation itself. The initial classical tuning of the boundary values of the couplings, relation (a) in Eq. (9), $\lambda_{\sigma}=9 \lambda_{m}^{2} / \lambda_{\phi}\left(1+\mathcal{O}\left(\lambda_{6}\right)\right)$, assumed to be true in the paper (for spontaneous breaking to exist), receives loop corrections of order $\mathcal{O}\left(\lambda_{j}\right)$. As a result, the tuning of the couplings, which enforces $V_{\min }=0$ at the loop level (demanded by scale symmetry), is $\mathcal{O}\left(\lambda_{j}\right)$ relative

\footnotetext{
${ }^{28}$ even if at classical level it was conserved.

${ }^{29}$ If $V$ contained mass terms, $\partial_{\mu} D^{\mu}$ would also contain a "classical" breaking of the scale symmetry term, $m^{2} \phi^{2}$.
}

to its tree-level case. More generally, in order $n$, the tuning is $\mathcal{O}\left(\lambda_{j}\right)$ relative to that in order $n-1$, i.e., at the level of the precision of the perturbation theory calculation in that order.

The consistency of the boundary values for the running couplings with some high-scale physics that must fix the value of $\langle\sigma\rangle$ should be investigated. This discussion requires one to extend this quantum calculation to the case of curved space-time while respecting this symmetry. The appropriate setup is in the context of the Brans-DickeJordan theory of gravity. As discussed in Ref. [40], in such a frame with nonminimal couplings, the dilaton (with derivative couplings) decouples and avoids "fifth force experiments." For investigations along this direction, see Refs. [28,29,32-38]. (Another possibility is to consider Einstein gravity, which breaks the scale symmetry discussed here. Then, scale symmetry is only an approximate symmetry, and the dilaton is a pseudo-Goldstone mode that acquires a small mass, and the vacuum energy is then nonzero).

\section{CONCLUSION}

We explored the possibility that scale symmetry is a quantum symmetry of the SM that is broken (only) spontaneously. Following previous developments on this idea, we considered the case of the classically scaleinvariant version of the SM that has vanishing tree-level mass for the Higgs $(\phi)$ and is extended by the dilaton $\sigma$ (the Goldstone mode of scale symmetry). The vev $\langle\sigma\rangle \neq 0$ breaks the scale symmetry spontaneously and dynamically generates a subtraction scale $\mu \sim\langle\sigma\rangle$ that is necessary for quantum calculations.

The classical scalar potential is dictated by the scale symmetry only and may contain nonpolynomial effective operators such as $\lambda_{6} \phi^{6} / \sigma^{2}, \lambda_{8} \phi^{8} / \sigma^{4}, \lambda_{10} \phi^{10} / \sigma^{6}, \lambda_{12} \phi^{12} / \sigma^{8}$, etc; these may always be Taylor expanded into a sum of infinitely many polynomial operators in fields fluctuations suppressed by powers of $\langle\sigma\rangle$ (which can be regarded as a physical cutoff of the theory), but in such a case, the manifest scale symmetry of the theory is lost.

The one-loop computation of the potential respected the scale symmetry of the classical Lagrangian. As a result, a scale-invariant one-loop potential for the Higgs and dilaton was obtained. The quantum potential had corrections from gauge and Yukawa interactions and also from the higherdimensional, nonpolynomial operators. The latter were included in the classical Lagrangian, and their couplings $\left(\lambda_{6}, \lambda_{8}, \lambda_{10}, \lambda_{12}\right.$, etc.) were one-loop renormalized with beta functions that we computed from the quantum potential. These beta functions were difficult to compute by other means and are an important result of this work. Tuning these couplings to zero at the tree level will not avoid the presence of their corresponding operators at the quantum level; these operators reemerge at the quantum level with a finite one-loop coefficient and as two-loop (scale-invariant) 
counterterms, due to the nonrenormalizability of theories with quantum scale invariance. The role of these (scaleinvariant) effective operators that capture the effects of an infinite series of polynomial operators deserves further study.

The quantum consistency of the calculation was verified by showing that the Callan-Symanzik equation of the quantum potential is respected in the presence of the nonpolynomial operators. We also showed the differences between the scale-invariant one-loop potential and its counterpart computed in the usual DR scheme $(\mu=$ constant $)$ that breaks scale symmetry explicitly, in the presence of nonpolynomial operators at tree level.

In quantum scale-invariant models, all mass scales are generated by vacuum expectation values of the fields, after spontaneous scale symmetry breaking; therefore, any mass hierarchy is not primary or fundamental but can be generated by a hierarchy of the (dimensionless) couplings of the theory. The vacuum energy is vanishing at the loop level in the case of spontaneously broken quantum scale symmetry, provided one coupling is a function of the rest; this ensures the flat direction exists. This can be arranged by one initial classical tuning, with subsequent, quantum tunings of $\mathcal{O}\left(\lambda_{j}\right)$ relative to the previous order. This picture is in contrast to the case in which the regularization explicitly breaks the classical scale symmetry of the action, leading to a different quantum theory (in which the minimum of the potential is nonzero).

It is possible to arrange a hierarchy $m_{\tilde{\phi}}^{2} \sim\langle\phi\rangle^{2} \ll\langle\sigma\rangle^{2}$ by choosing at the classical level an ultraweak coupling $\lambda_{m}$ between the SM and the hidden sector of the dilaton $\left(\left|\lambda_{m}\right| \ll \lambda_{\phi}\right)$ or by more elegant means (dynamics, etc.). This hierarchy is stable at the one-loop level, without additional tuning of the couplings and despite the presence of the nonrenormalizable operators mentioned. This UV behavior should survive to higher orders due to the spontaneous (i.e., soft) scale symmetry breaking.

\section{ACKNOWLEDGMENTS}

The work of D. M. G. was supported by a grant of the Romanian National Authority for Scientific Research (CNCS-UEFISCDI) under Project No. PN-II-ID-PCE2011-3-0607. The work of Z.L. was supported by the National Science Centre under Research Grant No. DEC2012/04/A/ST2/00099. The work of P. O. was supported by the National Science Centre, Poland, under Research Grant No. DEC-2016/21/N/ST2/03312.

\section{APPENDIX: ONE-LOOP BETA FUNCTIONS}

For convenience, we present the expressions of the beta functions found in the text:

$$
\begin{aligned}
\beta_{\lambda_{\phi}}= & \frac{1}{\kappa}\left[3\left(\frac{9}{4} g_{2}^{4}+\frac{3}{4} g_{1}^{4}+\frac{3}{2} g_{1}^{2} g_{2}^{2}-12 h_{t}^{4}\right)\right. \\
& \left.-4 \lambda_{\phi}\left(\frac{3}{4} g_{1}^{2}+\frac{9}{4} g_{2}^{2}-3 h_{t}^{2}\right)+4 \lambda_{\phi}^{2}+3 \lambda_{m}^{2}+96 \lambda_{m} \lambda_{6}\right], \\
\beta_{\lambda_{m}}= & \frac{2 \lambda_{m}}{\kappa}\left[\lambda_{\phi}+2 \lambda_{m}+\frac{1}{2} \lambda_{\sigma}-\left(\frac{3}{4} g_{1}^{2}+\frac{9}{4} g_{2}^{2}-3 h_{t}^{2}\right)\right], \\
\beta_{\lambda_{\sigma}}= & \frac{3 \lambda_{\sigma}}{\kappa}\left[\lambda_{\sigma}+4 \frac{\lambda_{m}^{2}}{\lambda_{\sigma}}\right], \\
\beta_{\lambda_{6}}= & \frac{3 \lambda_{6}}{\kappa}\left[6 \lambda_{\phi}-8 \lambda_{m}+\lambda_{\sigma}-2\left(\frac{3}{4} g_{1}^{2}+\frac{9}{4} g_{2}^{2}-3 h_{t}^{2}\right)\right], \\
\beta_{\lambda_{8}}= & \frac{2}{\kappa}\left[2 \lambda_{6}\left(28 \lambda_{6}+\lambda_{m}\right)-4 \lambda_{8}\left(\frac{3}{4} g_{1}^{2}+\frac{9}{4} g_{2}^{2}-3 h_{t}^{2}\right)\right], \\
\beta_{\lambda_{10}}= & \frac{10}{\kappa}\left[4 \lambda_{6}^{2}-\lambda_{10}\left(\frac{3}{4} g_{1}^{2}+\frac{9}{4} g_{2}^{2}-3 h_{t}^{2}\right)\right], \\
\beta_{\lambda_{12}}= & \frac{2}{\kappa}\left[3 \lambda_{6}^{2}-6 \lambda_{12}\left(\frac{3}{4} g_{1}^{2}+\frac{9}{4} g_{2}^{2}-3 h_{t}^{2}\right)\right] .
\end{aligned}
$$

[1] W. A. Bardeen, Report Nos. FERMILAB-CONF-95-391-T, C95-08-27.3.

[2] S. Weinberg, Gauge hierarchies, Phys. Lett. 82B, 387 (1979); G. 't Hooft, Naturalness, chiral symmetry, and spontaneous chiral symmetry breaking, in Recent Developments in Gauge Theories, edited by G.'t Hooft et al., NATO Advanced Study Institutes Series Vol. 59, (Plenum Press, New York, 1980), p. 135.

[3] F. Englert, C. Truffin, and R. Gastmans, Conformal invariance in quantum gravity, Nucl. Phys. B117, 407 (1976).

[4] M. Shaposhnikov and D. Zenhausern, Quantum scale invariance, cosmological constant and hierarchy problem, Phys. Lett. B 671, 162 (2009).
[5] M.E. Shaposhnikov and F. V. Tkachov, Quantum scale-invariant models as effective field theories, arXiv:0905.4857.

[6] R. Armillis, A. Monin, and M. Shaposhnikov, Spontaneously broken conformal symmetry: Dealing with the trace anomaly, J. High Energy Phys. 10 (2013) 030.

[7] C. Tamarit, Running couplings with a vanishing scale anomaly, J. High Energy Phys. 12 (2013) 098.

[8] D. M. Ghilencea, Manifestly scale-invariant regularization and quantum effective operators, Phys. Rev. D 93, 105006 (2016); One-loop potential with scale invariance and effective operators, Proc. Sci., CORFU2015 (2016) 040.

[9] F. Gretsch and A. Monin, Perturbative conformal symmetry and dilaton, Phys. Rev. D 92, 045036 (2015). 
[10] D. M. Ghilencea, Z. Lalak, and P. Olszewski, Two-loop scale-invariant scalar potential and quantum effective operators, Eur. Phys. J. C 76, 656 (2016).

[11] C. Wetterich, Cosmology and the fate of dilatation symmetry, Nucl. Phys. B302, 668 (1988).

[12] I. Bars, P. Steinhardt, and N. Turok, Local conformal symmetry in physics and cosmology, Phys. Rev. D 89, 043515 (2014) and references therein.

[13] W. Buchmuller and N. Dragon, Scale invariance and spontaneous symmetry breaking, Phys. Lett. B 195, 417 (1987); Dilatons in flat and curved space-time, Nucl. Phys. B321, 207 (1989).

[14] K. A. Meissner and H. Nicolai, Conformal symmetry and the standard model, Phys. Lett. B 648, 312 (2007); Effective action, conformal anomaly and the issue of quadratic divergences, Phys. Lett. B 660, 260 (2008).

[15] R. Foot, A. Kobakhidze, and R. R. Volkas, Electroweak Higgs as a pseudo-Goldstone boson of broken scale invariance, Phys. Lett. B 655, 156 (2007).

[16] H. Aoki and S. Iso, Revisiting the naturalness problemWho is afraid of quadratic divergences?, Phys. Rev. D 86, 013001 (2012).

[17] R. H. Boels and W. Wormsbecher, Spontaneously broken conformal invariance in observables, arXiv:1507.08162.

[18] R. Foot, A. Kobakhidze, K. L. McDonald, and R. R. Volkas, A solution to the hierarchy problem from an almost decoupled hidden sector within a classically scale invariant theory, Phys. Rev. D 77, 035006 (2008).

[19] B. Grinstein and P. Uttayarat, A very light dilaton, J. High Energy Phys. 07 (2011) 038.

[20] A. Farzinnia, H. J. He, and J. Ren, Natural electroweak symmetry breaking from scale invariant Higgs mechanism, Phys. Lett. B 727, 141 (2013).

[21] S. Iso, N. Okada, and Y. Orikasa, Classically conformal $B^{-}$L extended Standard Model, Phys. Lett. B 676, 81 (2009).

[22] S. Iso and Y. Orikasa, TeV Scale B-L model with a flat Higgs potential at the Planck scale-in view of the hierarchy problem, Prog. Theor. Exp. Phys. 2013, 023 B08 (2013).

[23] E. Gabrielli, M. Heikinheimo, K. Kannike, A. Racioppi, M. Raidal, and C. Spethmann, Towards completing the Standard Model: Vacuum stability, EWSB and dark matter, Phys. Rev. D 89, 015017 (2014).

[24] D. Gorbunov and A. Tokareva, Scale-invariance as the origin of dark radiation?, Phys. Lett. B 739, 50 (2014).
[25] Z. Chacko and R. K. Mishra, Effective theory of a light dilaton, Phys. Rev. D 87, 115006 (2013); B. Bellazzini, C. Csaki, J. Hubisz, J. Serra, and J. Terning, A Higgslike dilaton, Eur. Phys. J. C 73, 2333 (2013); A naturally light dilaton and a small cosmological constant, Eur. Phys. J. C 74, 2790 (2014).

[26] S. Fubini, A new approach to conformal invariant field theories, Nuovo Cimento A 34, 521 (1976).

[27] K. Endo and Y. Sumino, A scale-invariant Higgs sector and structure of the vacuum, J. High Energy Phys. 05 (2015) 030.

[28] P. G. Ferreira, C. T. Hill, and G. G. Ross, Scale-independent inflation and hierarchy generation, Phys. Lett. B 763, 174 (2016).

[29] P. G. Ferreira, C. T. Hill, and G. G. Ross, Weyl current, scale-invariant inflation and Planck scale generation, Phys. Rev. D 95, 043507 (2017).

[30] A. Kobakhidze, Quantum relaxation of the Higgs mass, Eur. Phys. J. C 75, 384 (2015).

[31] K. Allison, C. T. Hill, and G. G. Ross, Ultra-weak sector, Higgs boson mass, and the dilaton, Phys. Lett. B 738, 191 (2014).

[32] K. Kannike, M. Raidal, C. Spethmann, and H. Veermäe, Evolving Planck mass in classically scale-invariant theories, J. High Energy Phys. 04 (2017) 026.

[33] M. Shaposhnikov and D. Zenhausern, Scale invariance, unimodular gravity and dark energy, Phys. Lett. B 671, 187 (2009).

[34] D. Blas, M. Shaposhnikov, and D. Zenhausern, Scaleinvariant alternatives to general relativity, Phys. Rev. D 84, 044001 (2011).

[35] G. K. Karananas and M. Shaposhnikov, Scale invariant alternatives to general relativity. II. Dilaton properties, Phys. Rev. D 93, 084052 (2016).

[36] I. Oda, Higgs Mechanism in scale-invariant gravity, Adv. Stud. Theor. Phys. 8, 215 (2014).

[37] I. Oda, Conformal Higgs gravity, Adv. Stud. Theor. Phys. 9 , 595 (2015).

[38] I. Oda, Classically Scale-invariant B-L model and conformal gravity, Phys. Lett. B 724, 160 (2013).

[39] G. Marques Tavares, M. Schmaltz, and W. Skiba, Higgs mass naturalness and scale invariance in the UV, Phys. Rev. D 89, 015009 (2014).

[40] P. G. Ferreira, C. T. Hill, and G. G. Ross, No fifth force in a scale invariant universe, Phys. Rev. D 95, 064038 (2017). 